\title{
Extract of Caulis Spatholobi, a novel blocker targeting tumor cell-induced platelet aggregation, inhibits breast cancer metastasis
}

\author{
XI CHEN $^{1,2}$, QI LI ${ }^{2}$, XIAO-XI KAN ${ }^{2}$, YA-JIE WANG ${ }^{2}$, YU-JIE LI ${ }^{2}$, QING YANG $^{2}$, HONG-BIN XIAO $^{3}$, \\ YING CHEN $^{2}$, XIAO-GANG WENG ${ }^{2}$, WEI-YAN CAI ${ }^{2}$ and XIAO-XIN ZHU ${ }^{1,2}$ \\ ${ }^{1}$ Capital Medical University School of Traditional Chinese Medicine, Beijing 100069; ${ }^{2}$ Institute of Chinese Materia Medica, \\ China Academy of Chinese Medical Sciences, Beijing 100700; ${ }^{3}$ Key Laboratory of Separation Science for Analytical \\ Chemistry, Dalian Institute of Chemical Physics, Chinese Academy of Sciences, Dalian, Liaoning 116023, P.R. China
}

Received February 25, 2016; Accepted March 22, 2016

DOI: $10.3892 /$ or.2016.5184

\begin{abstract}
Metastasis of breast cancer is the vital step for malignant progression. During such a process, hematogenous metastasis is an indispensable approach for the dissemination of cancer cells. A platelet, contributes to hypercoagulable state, and is also identified the crucial factor in the coagulation system for supporting metastasis. Therefore, the relationship of a platelet and a tumor cell plays a critical role in tumor cell metastasis. Consequently, inhibiting tumor cell-induced platelet aggregation (TCIPA) is recongnized as a crucial target on suppression of tumor metastasis such as aspirin (ASA). Under such circumstance, here we report that, through dissociating the tumor-platelet (T-P) complex, 80\% ethanol extracts of Caulis Spatholobi (SET) successfully alleviated the hypercoagulation state, thereby reducing tumor metastasis and improving the prospects of survival in breast cancer cell model. Through MTT and anti-aggregation assay stimulated by ADP, we detected the optimum treatment time and the optimum dose of SET. By using confocal microscopy, we observed that SET can strongly block the formation of T-P complex in vitro. The result was further quantified and confirmed by the FACS analysis. The fluorescent value of T-P complex was obviously decreased in the drug-treated groups. In vivo, $4 \mathrm{~T} 1$ cells were injected through the mouse tail vein for dynamic visualization by small animal imaging system. The
\end{abstract}

Correspondence to: Dr Xiao-Xin Zhu, Institute of Chinese Materia Medica, China Academy of Chinese Medical Sciences, 16 Dongzhimen South Street, Dongcheng District, Beijing 100700, P.R. China

E-mail: zhuxiaoxin@icmm.ac.cn

Abbreviations: TCIPA, tumor cell-induced platelet aggregation; SET, $80 \%$ ethanol extracts of Caulis Spatholobi; ASA, aspirin; MMP, matrix metalloproteinase; SELP, selectin P; SELPLG, selectin P ligand; GPVI, glycoprotein VI; ITGA2B, integrin $\alpha 2 b$

Key words: $80 \%$ ethanol extracts of Caulis Spatholobi, tumor cell-induced platelet aggregation, breast cancer, metastasis, adhesion molecules metastatic intensity was quantified and the survival curve was analyzed. Additionally, general observation and hematoxylin and eosin (H\&E) staining of lung tissue was performed. SET exerted an obvious effect on the inhibition of metastasis and increasing the survival rate of mice. For the molecular mechanism study of anti-TCIPA, zymography and RT-PCR assay preliminarily revealed the molecular mechanism of SET in the regulation of P-T interaction. Collectively, through drug efficacy identification and pharmacological revealing, we have obtained a promising candidate for the interference of breast metastasis by suppressing TCIPA, which will be beneficial for clinical cancer treatment.

\section{Introduction}

Breast cancer is the most common cause of cancer mortality throughout the world. Taking America as an example, 231,840 new cases of breast cancer were estimated, among which, 40,290 breast cancer deaths are expected to occur among American females in 2015 (1).

The spreading and metastases formation of breast cancer are the vital procedure in the whole process of malignant disease, which is the main cause of cancer-related deaths. In addition, locally advanced metastases of breast cancer can be detected on lung, bone, liver and brain, developing into an incurable disease with adverse prognosis (2).

Furthermore, tumor metastasis to distant tissues is highly dependent on intravascular microenvironment (3), in which, platelets notably contribute to the metastatic process.

The basic physiological function of platelets is haemostasis and thrombosis. However, pro-metastatic activity of platelets have aroused wide concern (4). When platelets interacted with tumor cells in cancer patients, a collusive and detrimental pathological process occurs (5).

Platelets are activated by tumor cells during circulation, this interaction is defined as the switch of hypercoagulable state, which is recognized as tumor cell-induced platelet aggregation (TCIPA) (6). Based on the above phenomenon, abnormalities of platelets, once activated by tumors occur in the patients with malignancy, and thrombocytosis is diagnosed frequently. Furthermore, platelet physiological function is transferred to the pathological condition by degrees. The 
process is associated with coagulation formation and cancer metastasis (7). On the contrary, following activation, abnormal platelets are recruited to the tumors and their microenvironment. After that, tumor is escorted by the platelets to each essential part of tumor hematogenous metastasis (8). By virtue of platelet abnormalities, thrombi forms within a blood vessel, then, thrombosis is developed, which is a hallmark of most cancer metastasis.

Sequentially, in the first stage of metastasis, platelets facilitate tumor invasion to adjacent blood vessels. Then, in the circulation, platelets are enriched in the vicinity of tumor cells, forming a tumor-platelet (T-P) clot, which shield tumor cells escaping from the immune response of T-cell and surveillance of NK cells, and prevent damage of shear stress resulting from blood flow. Next, the clot, consisting of platelet and tumor, is arrested at a distant metastatic organ. Eventually, metastatic colonization adapt to the foreign microenvironment for survival and development after extravasation (9).

During the progress of TCIPA, two types of mechanisms on (T-P) interaction have been recognized. The first, direct physical binding of platelet receptors and corresponding tumor-derived ligands leads to the activation of platelet and the enhancement of platelet adhesion and aggregation. The binding is not only a simple cell to cell cohesion, but also a kind of high-affinity specific interaction. The tissue factor (TF), glycoprotein VI (GPVI) and glycoprotein Ib $\alpha$ (GPIb $\alpha)$ expressed on tumor cells are respectively bound to the peroxisome activation receptor (PAR) and platelet integrin $\alpha \operatorname{IIb} \beta 3$. Notably, the selectin $\mathrm{P}$ on the surface of the platelets is tethered to selectin P ligand (SELPLG) protein on tumor cells (10). Secondly, countless soluble factors secreted from activated platelets and tumor cells indirectly remodel a suitable and common microenvironment, in which, the ability of tumor cells to survive and metastasize is strengthen. For example, vascular endothelial growth factor (VEGF), transforming growth factor- $\beta 1$ (TGF- $\beta 1$ ) and platelet-derived growth factor (PDGF) affects the function of cancer-related inflammation, angiogenesis, tumor growth and metastasis (10). Leading by above analysis, TCIPA was the critical point of cancer progress, shown to interrelate closely with metastasis. Thus, TCIPA-targeted therapy should be a feasible strategy of cancer treatment such as aspirin (ASA).

In traditional medicine, certain drugs have been discovered that affect anti-aggregation, such as Caulis Spatholobi. Caulis Spatholobi, the vine stem of Spatholobus suberectus Dunn., have been used widely as a kind of drug for invigorating blood circulation and eliminating stasis. However, there is no report on the efficacy of $80 \%$ ethanol extracts of Caulis Spatholobi (SET) blocking TCIPA, and eventually inhibiting tumor metastasis. Overall, we identified the optimal treatment dose and time of SET, discuss the anti-TCIPA and anti-metastasis efficacy of SET in vitro and in vivo, and revealed the preliminary mechanism of SET for inhibiting TCIPA. Thus, our results showed that SET could effectively contribute to TCIPA as a potential target for anti-metastatic therapy.

\section{Materials and methods}

Cell culture. The murine mammary carcinoma cell stably expressing the firefly luciferin gene (4T1-luc) and non-labeled
4T1 cells were obtained from Dr Ganlin Zhang, Beijing Hospital of Traditional Chinese Medicine (Beijing, China). The cell line was cultured in RPMI-1640 medium (Gibco, Grand Island, NY, USA) and supplemented with $10 \%$ fetal bovine serum (HyClone, Logan, UT, USA), $100 \mathrm{mg} / \mathrm{ml}$ streptomycin and $100 \mathrm{U} / \mathrm{ml}$ penicillin at $37^{\circ} \mathrm{C}$ in a humidified atmosphere with $5 \% \mathrm{CO}_{2}$.

Reagents. SET was extracted by the Dalian Institute of Chemical Physics, Chinese Academy of Sciences (Dalian, China). ASA was purchased from the National Institutes for Food and Drug Control (Beijing, China).

Extraction and preparation of drug. Two kilograms of Caulis Spatholobi were decocted thrice with anhydrous ethanol. The liquids were merged and concentrated to constant weight on a rotary vacuum evaporator. Further purification of the extracts (20 g) was performed by HPD-100 macroporous adsorbent column chromatography eluted with different concentrations of alcohol. Respectively, the fractions of 20, 40, 60, 80 and 100\% alcohol were concentrated to constant weight on a rotary vacuum evaporator and crushed into powder before experiments. The extracts were named SE1, SE2, SE3, SET, and SE4, respectively.

In vitro cytotoxicity of the Caulis Spatholobi extracts. The screening of cytotoxicity was assessed by MTT (Amresco, USA) assay as previously described (11). Briefly, the 4T1 cells were plated into a 96 -well cell culture cluster at $5 \times 10^{3}$ per well. Then, the cells were treated with SE1, SE2, SE3, SET, and SE4 in different concentration, respectively, for 24, 48, 72 and $96 \mathrm{~h}$. Another experiment was performed using SET as treatment, which was identified by the above preliminary test. The method was conducted as mentioned above, however, the time of drug treatment was adjusted to 3, 6, 12 and $24 \mathrm{~h}$. At mentioned time points, MTT was added to each well till the final concentration of $0.5 \mathrm{mg} / \mathrm{ml}$, and the cells were incubated at $37^{\circ} \mathrm{C}$ for $4 \mathrm{~h}$. A total of $150 \mu \mathrm{l}$ of dimethyl sulfoxide (DMSO) was replaced in each well instead of medium with MTT. With fully mixing, the absorbances of solution were determined in $570 \mathrm{~nm}$ performed a microplate reader (Molecular Devices, USA). The inhibitory rate of 4T1 cells was calculated as follows. Inhibitory rate $=(1-$ mean experimental absorbance $/$ mean negative control absorbance) x $100 \%$. Half maximal inhibitory concentration was calculated as previously described (12).

Collection of blood and preparation of platelet. Fresh blood was obtained as previously described (13). In brief, blood was obtained by retro-orbital venous plexus sampling, which was collected into an Eppendorf tube mixed with acid citrate dextrose solution (38 $\mathrm{mM}$ citric acid/75 $\mathrm{mM}$ trisodium citrate/100 $\mathrm{mM}$ dextrose). The blood was centrifuged at $280 \mathrm{x}$ g for $8 \mathrm{~min}$ at room temperature, supernatant containing plasma and buffy coat were transferred to a new tube, centrifuged at $280 \mathrm{x} \mathrm{g}$ for $4 \mathrm{~min}$. Then, platelet-rich plasma (PRP) was collected into a fresh tube and prepared for study.

ADP-stimulated platelet aggregation. The different doses of SET were designed as low, medium, high $(20,40$ and $80 \mu \mathrm{g} / \mathrm{ml})$ groups and ASA $(300 \mu \mathrm{M})$ as positive control group compared with negative control. The following study in vitro was based 
on identical methods. The platelet aggregation was measured by whole blood aggregometers (Chrono-Log, Havertown, PA, USA) (14). Briefly, $500 \mu 1$ of whole blood with equivalent volume of normal saline (NS) was placed in aggregometer, incubated for $10 \mathrm{~min}$ at $37^{\circ} \mathrm{C}$ with stirring at $1,200 \mathrm{rpm}$. The samples were treated by SET as inhibitors. ADP $(10 \mu \mathrm{M})$ was added to the mixture as activator for platelet aggregation. Finally, the rates of aggregation were recorded through a curve. To determine the optimal dose, the different concentrations of SET were used to inhibit the platelet aggregation induced by ADP.

Tumor cell-induced platelet aggregation. As described by the previous method, PRP was used within $2 \mathrm{~h}$ after centrifugation. Next, carboxyfluorescein diacetate-succinimidyl ester (CFDA-SE; Beyotime, Shanghai, China), a sort of cell fluorescent label, was incubated with PRP at a final concentration of $5 \mu \mathrm{M}$ for $10 \mathrm{~min}$. The labeled platelets were washed by Tyrode's solution twice, then, resuspended with fresh Tyrode's solution. The next section of test was performed after at least $1 \mathrm{~h}$. The TCIPA was detected by confocal microscopy and flow cytometry assay as previously described (15). For the microscopic observation of TCIPA, briefly, $1 \times 10^{5}$ cells $/ \mathrm{ml}$ 4T1 cells, pre-treated by SET and ASA, were plateted in the confocal dish until the logarithmic phase. Fluorescently labeled platelets were placed into the dish and incubated with tumor cells for $30 \mathrm{~min}$ at $37^{\circ} \mathrm{C}$. The non-aggregated platelets were removed. T-P cell aggregation was visualized by confocal microscopy (Olympus, Tokyo, Japan). For flow cytometry assay, $1 \times 10^{5}$ pre-treated $4 \mathrm{~T} 1$ cells were incubated with $200 \mu \mathrm{l}$ of platelet preloaded with CFDA-SE for $10 \mathrm{~min}$. A final concentration of $2 \%$ paraformaldehyde was added as a fixative stored at $4^{\circ} \mathrm{C}$, prepared to flow cytometry assay.

In vivo metastatic seeding assay. For lung seeding and metastasis analysis, the mouse model of pulmonary metastasis was established by vein tail injection as previously described (16). Briefly, $2 \times 10^{4} 4 \mathrm{~T} 1$-luc cells in $100 \mu \mathrm{l}$ of NS were injected into 8 -week-old female Balb/c mice by vein tail. Simultaneously, mice were administrated with different doses of SET (2.2, 4.4 and $8.8 \mathrm{mg} / \mathrm{kg})$. ASA $(22.5 \mu \mathrm{mol} / \mathrm{kg})$ was used as positive control drug compared with model group (tumor only). Imaging assay was conducted after injection of tumor cells and daily therapy. Mice were anesthetized by isoflurane gas, then visualized by using small animal in vivo imaging system (Kodak, USA) 10 min after injection of luciferin. Until the mice of model group died, mice were sacrificed and the lungs were collected into $4 \%$ paraformaldehyde for observation and hematoxylin and eosin (H\&E) staining (17) using a microscope (Olympus). Survival curve was employed to evaluate overall survival. The study was approved by the Ethics Committee of Beijing Administration Rule of Laboratory Animal, Beijing, China.

Sample preparation of TCIPA for zymography. The method of cell plating and drug treatment was measured as mentioned above. In the logarithmic phase, the 4T1 cells cultured in FBS-free medium were incubated with $200 \mu \mathrm{l}$ PRP, or not, for $10 \mathrm{~min}$. Then, the reaction was terminated and samples were collected by centrifugation at $900 \mathrm{x}$ g for $10 \mathrm{~min}$. Supernatant was stored at $-80^{\circ} \mathrm{C}$ until the activities of Matrix metalloproteinase (MMP)-2 and -9 were detected by zymography.
Zymography assay. Gelatin zymography was used to detect the activity of MMP-2 and -9 in the platelet supernatants as previously described (18). In brief, samples of TCIPA were separated by $10 \%$ SDS-PAGE with copolymerized gelatin $(0.2 \%$; Sigma Chemical Co., St. Louis, MO, USA) with a substrate for gelatinolytic proteases. After electrophoresis, the gels were washed with $2.5 \%$ Triton $\mathrm{X}-100$ for 20 min repeated three times, and then incubated for $48 \mathrm{~h}$ at $37^{\circ} \mathrm{C}$ in incubating buffer $\left(25 \mathrm{mM}\right.$ Tris $\mathrm{HCl}, 0.9 \% \mathrm{NaCl}, 5 \mathrm{mM} \mathrm{CaCl}_{2}$ and $0.05 \%$ $\left.\mathrm{Na}_{3} \mathrm{~N}, \mathrm{pH} 7.5\right)$. The conditioned medium of $4 \mathrm{~T} 1$ cells was used as control. After $72 \mathrm{~h}$ reaction, gels were fixed and stained in $0.1 \%$ Coomassie blue R-250 (Sigma Chemical Co.), $40 \%$ methanol and $10 \%$ acetic acid for $1 \mathrm{~h}$ and then discolored in $8 \%$ acetic acid with $4 \%$ methanol. The gelatinolytic activities were presented by separate bands.

RT-PCR assay. The methods of cell plating and drug treatment were measured as indicated above. Total RNA was isolated by TRIzol reagent (Life Technologies, Grand Island, NY, USA). The detailed procedure of RT-PCR was measured by RevertAid First Strand cDNA Synthesis kit (Thermo Fisher Scientific, Waltham, MA, USA) (19). According to the kit instruction, $1 \mu \mathrm{g}$ total RNA was performed to the following reverse transcriptase in a final volume of $20 \mu \mathrm{l}$ with AMV reverse transcriptase. Subsequently, several specific primers were used to amplify cDNAs of selectin P (SELP) forward, 5'-CGA GCCCAACAACAAGAA-3' and reverse, 5'-GGGTAGCAG GAGCAGGTAT-3'; selectin P ligand (SELPLG) forward, 5'-CTGTCACTGAGGCAGAGTCG-3' and reverse, 5'-TGA GCAGCCACGGTGTTG-3'; GPVI forward, 5'-CCTTCCATC TTACCCACA-3' and reverse, 5'-CTGCTGAAAGCCAAGT TAT-3'; integrin $\alpha 2 b$ (ITGA2B) forward, 5'-TGACAGGGA GCAGGAAGA-3' and reverse, 5'-GCTGGGCGTGGATAG TTT-3'. GAPDH (20) forward, 5'-GTGGATATTGTTGCCA TCA-3' and reverse, 5'-ACTCATACAGCACCTCAG-3' was compared with each experiment as internal control. The PCR products were analyzed by agarose gel electrophoresis and ethidium bromide staining.

Statistical analysis. The values of data were presented as means \pm standard deviation. Statistical significance was determined by one-way analysis of variance followed by LSD post hoc test using SPSS Statistics 17.0. $\mathrm{P}<0.05$ was considered to be statistically significant, ${ }^{*} \mathrm{P}<0.05,{ }^{* *} \mathrm{P}<0.01$, ${ }^{* * *} \mathrm{P}<0.001$.

\section{Results}

Screening and dose identification of SET in $4 T 1$ cells. Taking breast cancer as cell model, we screened the proliferation inhibitory effect of SET in 4T1 cells by MTT assay. The proliferation of 4T1 cells was inhibited by SE1, SE2, SE3, SET, SE4 in a time- and dose-dependent manner, respectively. Taking the $50 \%$ inhibition rate as the cytotoxic criteria, to avoid the non-selective cytotoxicity, various doses of tested drugs and prolonged treatment times were designed and the cell viability was evaluated (Fig. 1A-E). The result showed little cytotoxicity for the tested extracts with low doses when they are treated for $<48 \mathrm{~h}$. In contrast, in long-term treatment ( $>48 \mathrm{~h})$, the non-selective cytotoxicity can be clearly detected in all of 

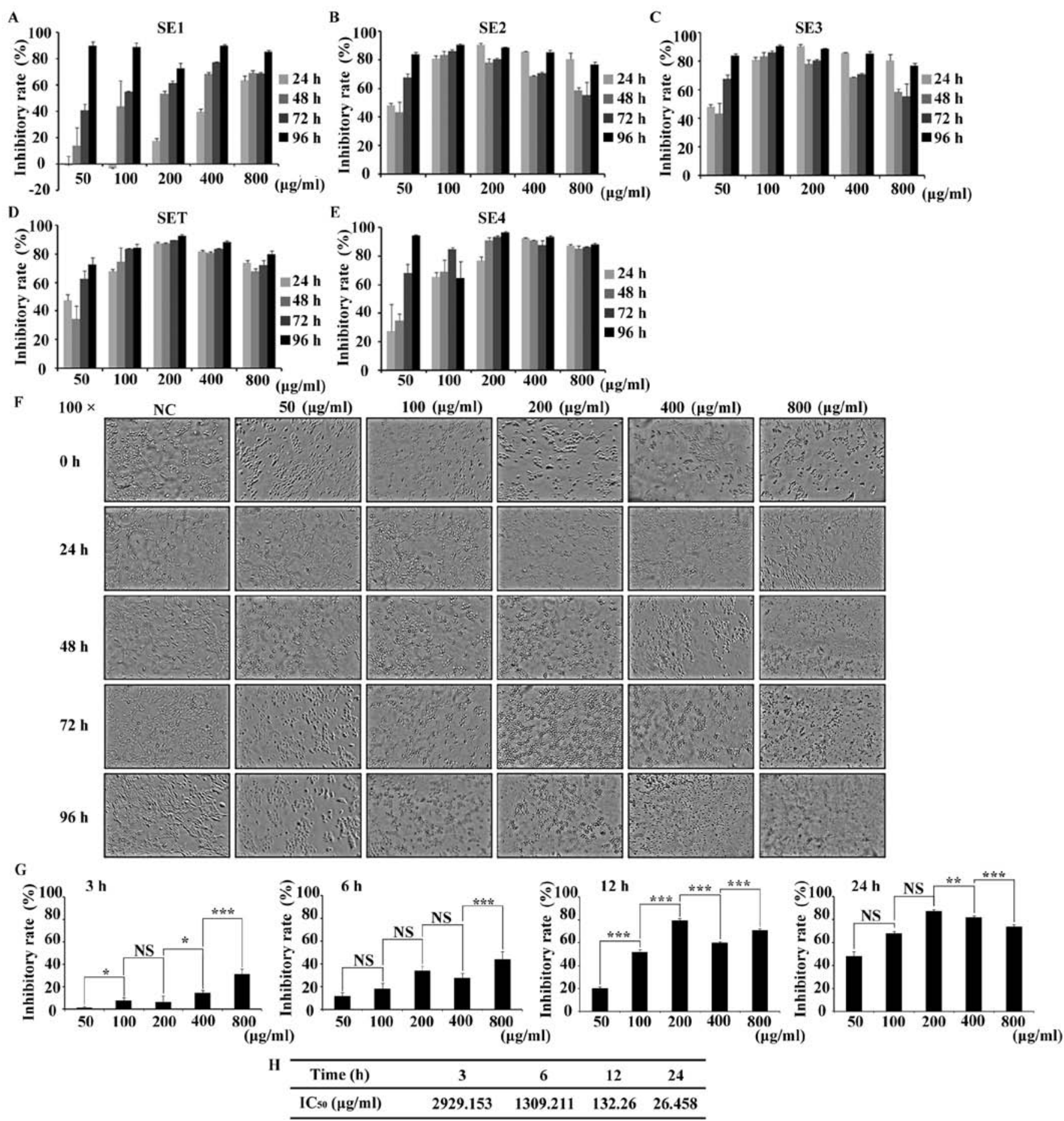

Figure 1. The screening of cytotoxicity of $80 \%$ ethanol extracts of Caulis Spatholobi (SET) in the 4T1 cell line. (A-E) Different ethanol extract of Caulis Spatholobi inhibited the 4T1 cell proliferation. The cell viability was greatly suppressed by (A) SE1, (B) SE2, (C) SE3, (D) SET and (E) SE4 treatment for $>48 \mathrm{~h}$ and $100 \mu \mathrm{g} / \mathrm{ml}$ in a dose- and time-dependent manner. (F) The morphological observation (x100) of SET-treated 4T1 cells for 3, 6, 12 and $24 \mathrm{~h}$. Within $6 \mathrm{~h}$, SET had little non-selective cytotoxicity in cell proliferation except at the dose of $800 \mu \mathrm{g} / \mathrm{ml}$. (G) The statistics of inhibition rate of SET, which was consistent with earlier results $(\mathrm{F}) .(\mathrm{H}) \mathrm{IC}_{50}$ value of each time point could be estimated and it reduced sharply with the time extension. Data are presented as means $\pm 95 \% \mathrm{CI},{ }^{*} \mathrm{P}<0.05,{ }^{* *} \mathrm{P}<0.01,{ }^{* * *} \mathrm{P}<0.001, \mathrm{~N}=3$, one-way ANOVA test and means \pm standard error of triplicates. NS, no significant difference.

the high dose extracts $(>400 \mu \mathrm{g} / \mathrm{ml})$. Based on preliminary analysis, in order to determine a drug that interacts with $4 \mathrm{~T} 1$ cells with low non-selective cytotoxicity, SET was selected for more detailed testing. The morphological analysis revealed that the SET treatment was time- and dose-dependent (Fig. 1F). Such influence of SET was further quantified by MTT assay. As shown in Fig. 1G after 6-h treatment, cell inhibition rate approached or exceeded 50\%, which implied that $<6 \mathrm{~h}$ of treatment is optimal for efficacy study. Moreover, $\mathrm{IC}_{50}$ values calculated at $3,6,12$ and $24 \mathrm{~h}$ were 2929.153 , $1309.211,132.26$ and $26.458 \mu \mathrm{g} / \mathrm{ml}$ (Fig. 1H), respectively. Thus, the safe and effective concentrate interval $(<400 \mu \mathrm{g} / \mathrm{ml})$ and treatment time $(6 \mathrm{~h})$ of SET were determined for further research 

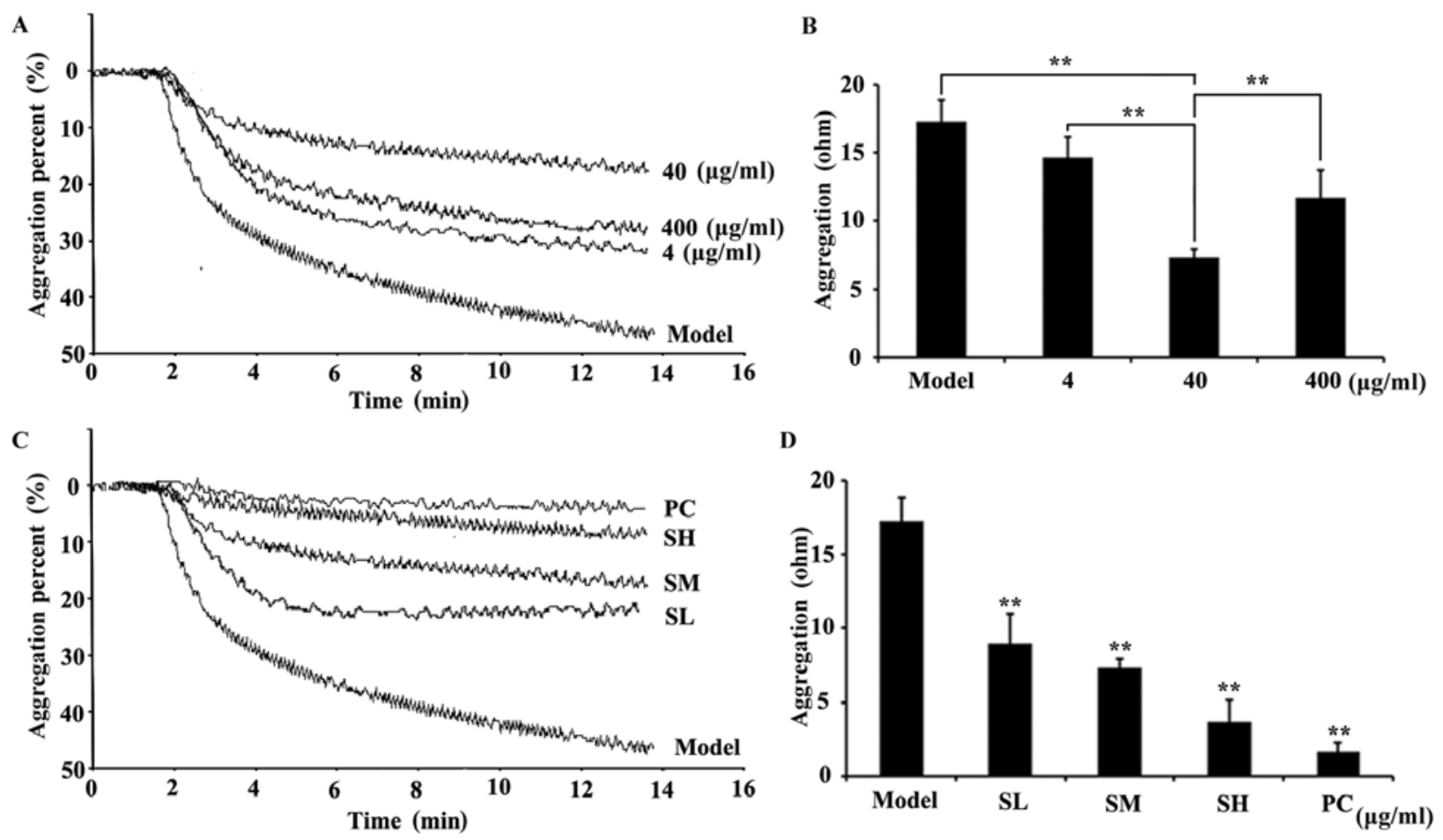

Figure 2. The 80\% ethanol extracts of Caulis Spatholobi (SET) inhibits platelet aggregation induced by ADP. (A and B) The curve of aggregation induced by $10 \mu \mathrm{M}$ ADP. Each concentration of SET showed a common efficacy of anti-aggregation. Notably, the $40 \mu \mathrm{g} / \mathrm{ml}$ of SET showed stronger efficacy compared with model group. (C and D) SET, $20 \mu \mathrm{g} / \mathrm{ml}$ (SL) and $40 \mu \mathrm{g} / \mathrm{ml}$ (SM), inhibited the aggregation dose-dependently. A total of $80 \mu \mathrm{g} / \mathrm{ml}$ (SH) of SET was the optimum dose of SET for anti-aggregation. Aspirin (ASA) was used for positive control drug (PC). Data are presented as means $\pm 95 \% \mathrm{CI},{ }^{* *} \mathrm{P}<0.01, \mathrm{~N}=3$, one-way ANOVA test.

Effects of SET on the suppression of platelet aggregation induced by $A D P$. Inspired by the traditional anticoagulation efficacy of Caulis Spatholobi, by using the active extract (Fig. 1), we further questioned whether our newly prepared extract (SET) affects the inhibition of platelet aggregation induced by ADP. The platelet aggregation assay results clearly showed that, in the model group, the platelet aggregation could be obviously induced in response to ADP stimulation. In contrast, the SET treatment, with 4, 40 and $400 \mu \mathrm{g} / \mathrm{ml}$ doses, could largely reverse such changes, as represented by the decrease of resistance values $(\mathrm{Ohm})$. This result suggested that the coagulation cascade was significantly blocked by SET. Notably, the anticoagulation activity of SET exhibits in a typical dose-dependent manner. SET at $40 \mu \mathrm{g} / \mathrm{ml}$ exerted the strongest efficacy (Fig. 2A and B). Taking these data into account, the $40 \mu \mathrm{g} / \mathrm{ml}$ dose interval was chosen for further efficacy study. A more detailed dose-dependent efficacy study was also performed. The treatment of SET, with 20, 40 and $80 \mu \mathrm{g} / \mathrm{ml}$ doses, significantly inhibited platelet aggregation dose-dependently. Compared with the model group, the SET treated groups showed an obviously decrease of platelets aggregation (Fig. 2C and D). Consequently, SET was able to inhibit the activation induced by ADP. However, the role of SET in the interaction between tumor and platelet and the inhibitory effects on the TCIPA are still unknown.

Effects of SET on the inhibition of TCIPA. To further reveal the efficacy of SET in vitro, we focused on TCIPA, during which SET exerted the effectiveness on the adhesion of platelets to
4T1 cells. In this study, 4T1 cells were incubated with platelets stained with fluorescent probe CFDA-SE. In the presence or absences of SET, non-aggregated platelets were totally washed and removed. Then, the stained platelets were observed in the vicinity of tumor cells by using a confocal microscope (Fig. 3A). The result showed that the quantity and intensity of green fluorescence in model group was much clearer. In drug-treated groups, fluorescence intensity was markedly decreased, which represents the reduced level of TCIPA. Additionally, such effect was further quantified via flow cytometry analysis. In the absence of SET, TCIPA could be clearly detected as measured by the elevated positive fluorescent ratio. On the contrary, in the presence of SET, the fluorescent positive rate was decreased obviously (Fig. 3B). Therefore, SET was able to efficiently inhibit the platelet aggregation induced by tumor cells. Anti-TCIPA therapy may be a potential drug target for SET that regulates the process of tumor metastasis in the blood circulation.

In vivo anti-metastatic efficacy of SET in mouse tail vein injection model. To confirm the efficacy of SET in vivo, excluding the effect of SET on the tumor in situ, we selected the tail vein injection model for detecting and analyzing the intensity of lung metastasis of breast cancer. Briefly, for experiments, drug administration was initiated 2 days before modeling with low-, medium- and high-dose of SET, once daily. The ASA was also used for positive control. Metastatic process was measured by small animal in vivo imaging system. The bioluminescence imaging on the lung was first observed on the 21st day after tumor cell injection in the modeling group. 


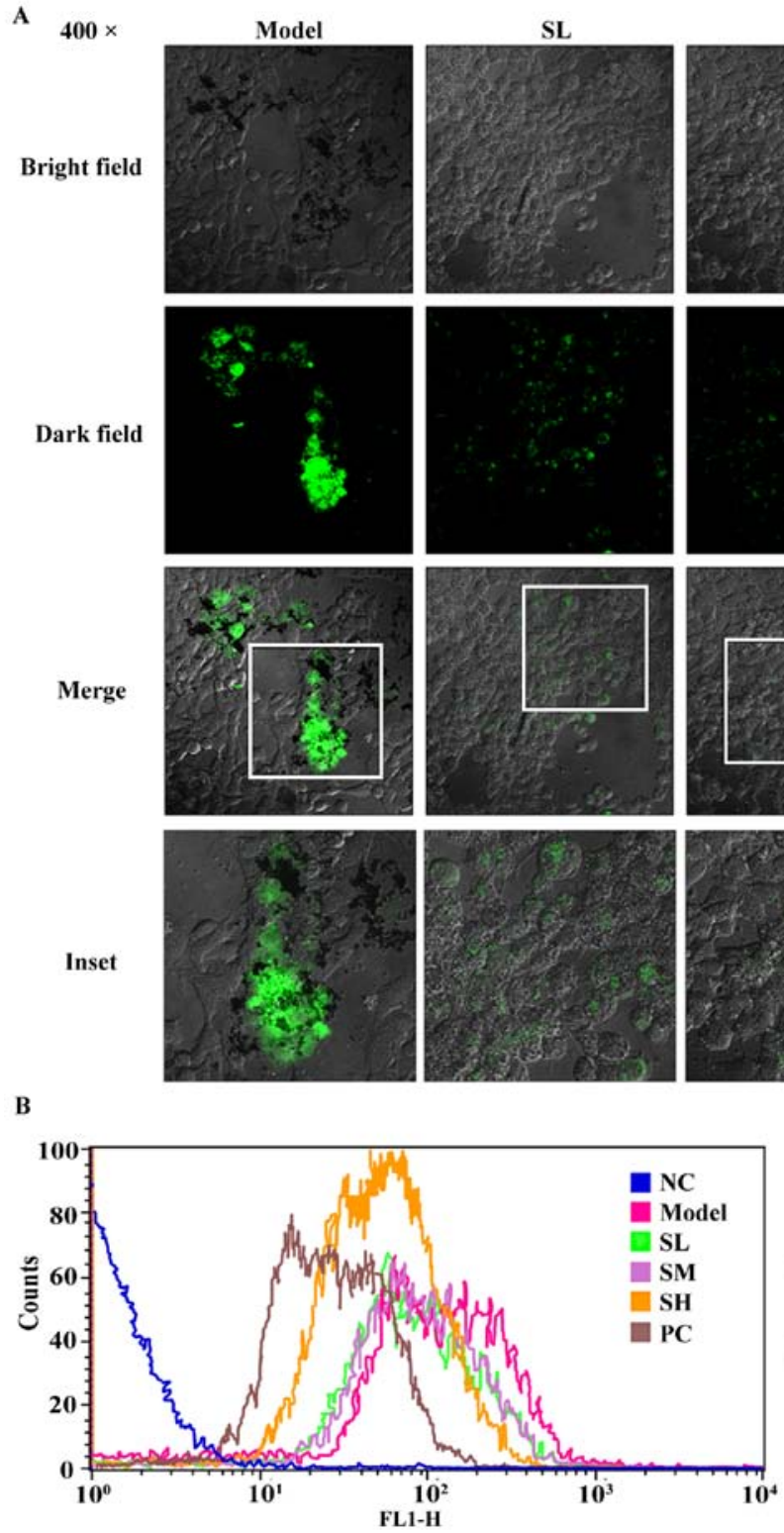

SM
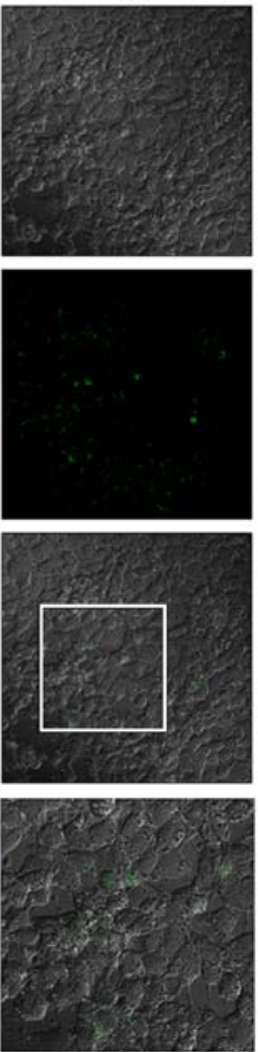

SH
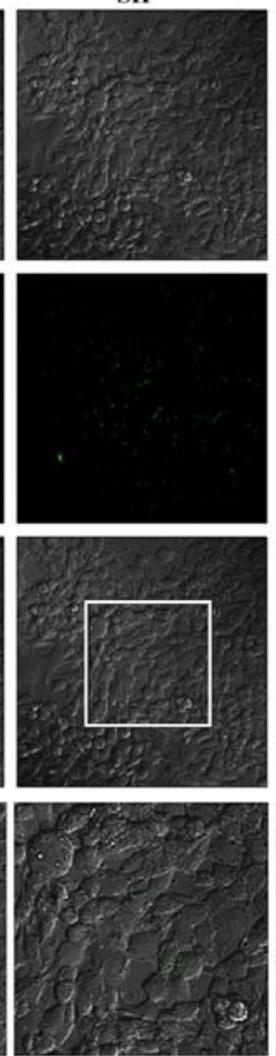

PC
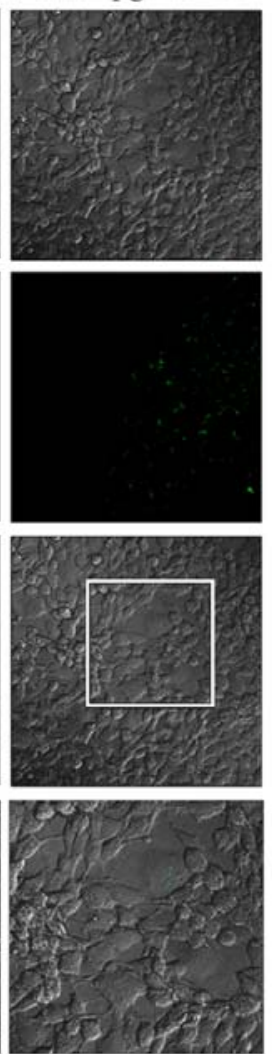

B

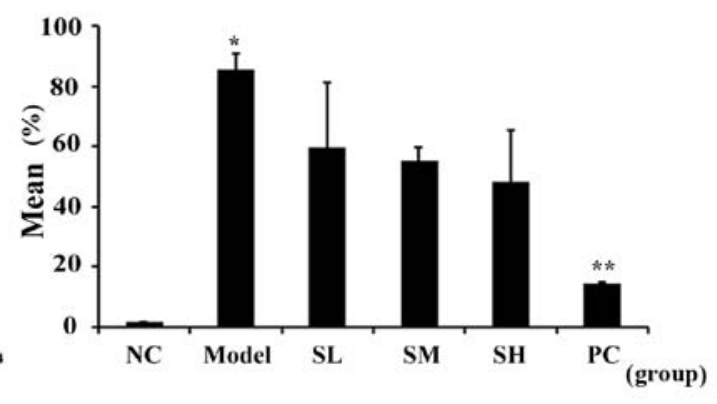

Figure 3. The $80 \%$ ethanol extracts of Caulis Spatholobi (SET) efficiently blocks the association of platelets with 4T1 cell in vitro. (A) The 4T1 cell-induced platelet aggregation was observed by confocal microscopy (x400). The bright field micrograph showed that the $4 \mathrm{~T} 1$ cells were in good condition. The cell count and viability of each group were approximately in the same level. In the dark field micrograph, the platelet stained green fluorescence could be seen clearly in model group compared with the drug-treated group. The merged image showed that the platelet aggregations were in the vicinity of tumor cells. The inset micrograph showed the details that the tumor-platelet aggregation was blocked. (B) The changes in platelets aggregated with tumor cells were demonstrated in the presence or absence of SET by flow cytometry. SET reduced the adhesion between tumor and platelet with a tendency of increase in a dose-dependent manner. Data are presented as means $\pm 95 \% \mathrm{CI},{ }^{*} \mathrm{P}<0.05,{ }^{* *} \mathrm{P}<0.01, \mathrm{~N}=3$, one-way ANOVA test.

As shown in Fig. 4A, mice treated with SET were visualized and showed less metastases compared to modeling mice. For quantitative analysis, the photon statistics measurements of coherent fields are shown in Fig. 4B. They are negatively influenced in a significant dose-dependent manner. To further analyze the intensity of metastasis in the lung, we performed general observation and $\mathrm{H} \& \mathrm{E}$ staining assay (Fig. 5A and B). Consistently, both of the results confirmed the obvious inhibition of lung metastasis in SET-treated mice. Moreover, the mice in the modeling group showned a poor survival rate compared with SET-treated mice (Fig. 4C). Thus, an obvious anti-metastasis efficacy of SET was evaluated. Consequently, SET has the possibility to be an anti-metastatic drug by targeting TCIPA in breast cancer.
MMP-2 and -9 released during TCIPA. In recent studies, MMP-2 and -9 were proven to play an important role in the association with platelet and tumor cell (21). Leading by this, zymography assay was measured to detect whether MMP-2 and -9 were released or not during TCIPA. As shown in Fig. 5C, we found that MMP-2 and -9 were released during TCIPA. However, the gray scale value of zymographic bands in each group had no statistical difference which indicated that SET failed to regulate the secretion of MMP-2 or -9 during T-P interaction. Consequently, the efficacy of SET on the inhibition of TCIPA was MMP-independent.

To investigate the exact mechanism of SET for blocking the association between platelets and tumor cells, we focused the efficacy of SET in the regulation of adhesive interaction 
A

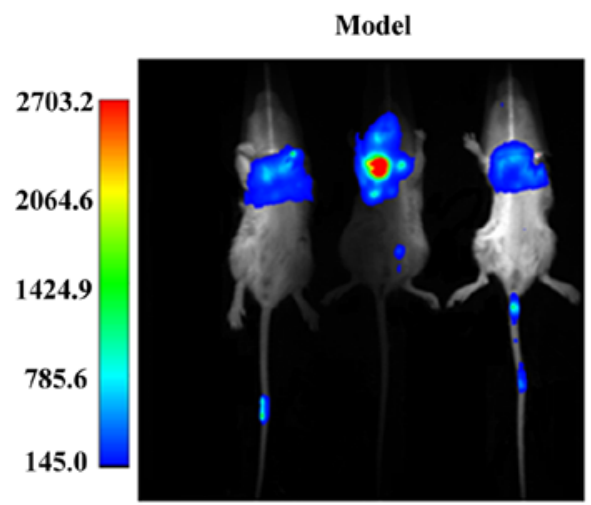

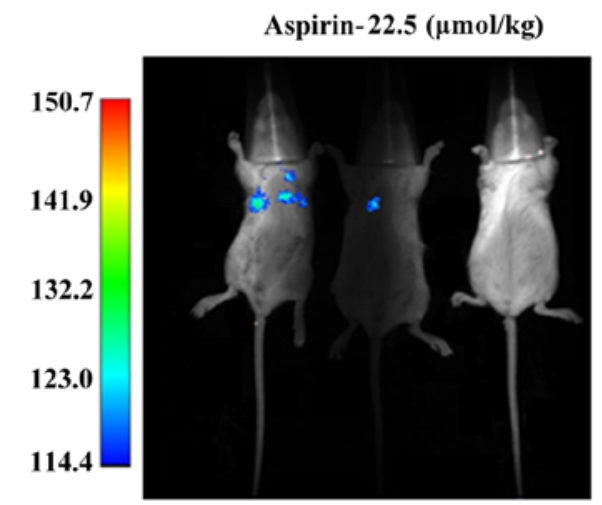
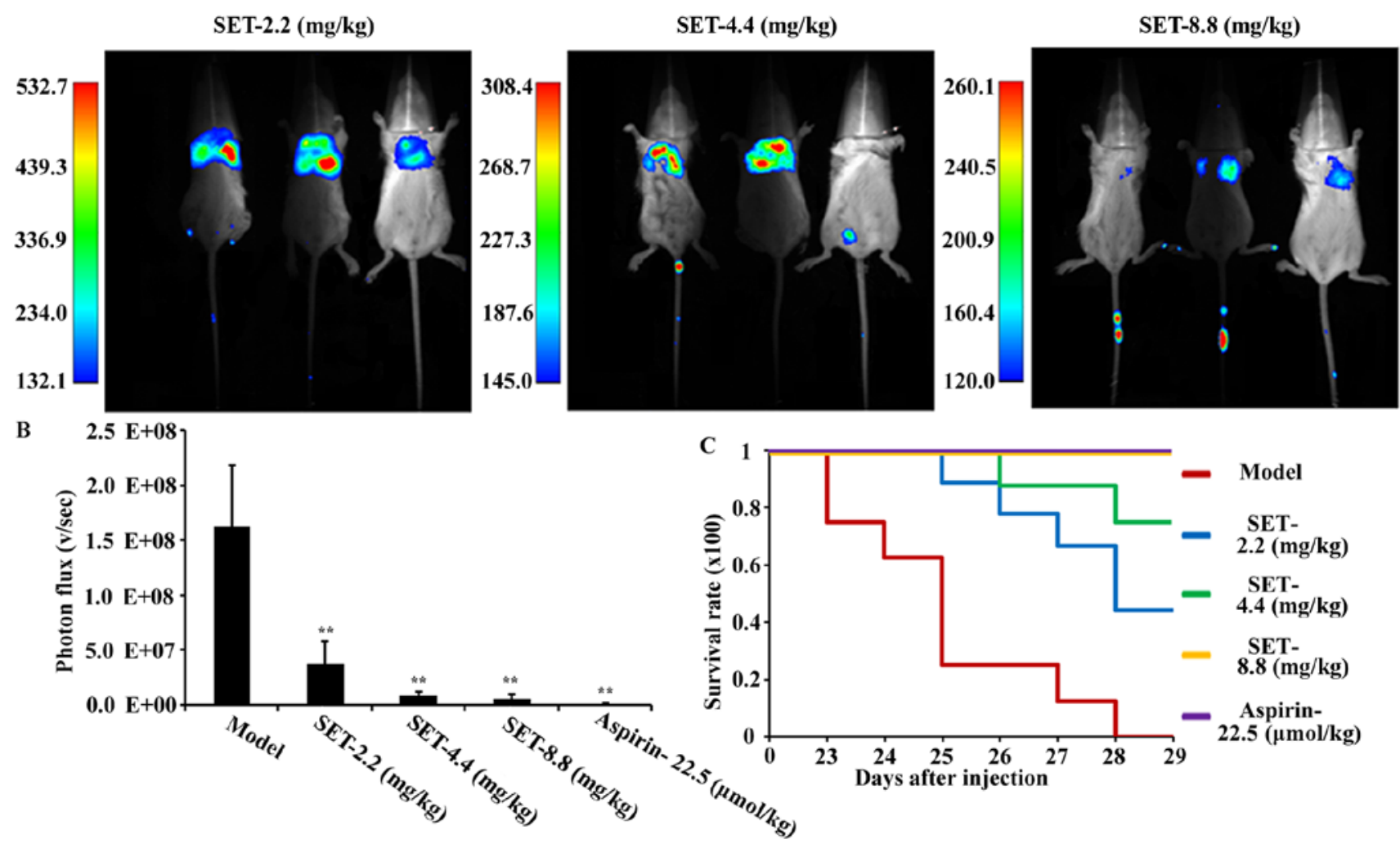

Figure 4. Efficacy of $80 \%$ ethanol extracts of Caulis Spatholobi (SET) inhibits breast cancer metastasis in vivo. (A and B) The anti-metastasis efficacy of SET was identified in vivo by small animal imaging system. The images were (A) collected and (B) evaluated by the measurement of total photons of metastases. The images in model group show a strong metastasis in the absence of SET after tumor vein tail injection. The anti-metastasis efficacy of SET was dose-dependent. (C) Survival curve showed that SET could evidently suppress the process of metastasis and improve the rate of survival. Data are presented as means $\pm 95 \% \mathrm{CI}$ from eight mice. ${ }^{* *} \mathrm{P}<0.01, \mathrm{~N}=8$, one-way ANOVA test.

between platelets and tumor cells. As commonly recognized, the key adhesive molecules such as SELP, SELPLG, GPVI, ITGA2B involved in tumor metastasis played a crucial role in TCIPA (22). Therefore, we detected the expression of above molecules as measured by RT-PCR assay. As expected, SET significantly inhibited SELP, GPVI, and ITGA2B expression compared with model group (Fig. 5D).While through analysis of RNA bands and measurement of the intensity of gray scale value, the expression of SELPLG could not be regulated by SET efficiently. We then identified that the expression of SELP, GPVI, and ITGA2B were suppressed by SET during TCIPA. Consequently, the mechanism of SET to inhibit TCIPA and reduce metastasis may relate with regulating the cell adhesion molecules and then suppressing adhesion of tumor cells and platelets.

\section{Discussion}

Hypercoagulable state is recognized as a pathological process whose main feature is an imbalance between coagulation and anticoagulation. The over-activated coagulation or the excessive suppression of anticoagulation both could contribute to the abnormality.

In 1865, it was found for the first time that cancer patients had abnormality of blood coagulation, and were at high risk for developing venous thrombosis (23). Substantial research proved that blood viscosity in patients with advanced tumor kept at a high level, and was characterized by increasing of fibrinogen, coagulation factor, especially platelets. The increase of blood viscosity in circulation is affected by two synergetic sides. On the one hand, the coagulation system 
A

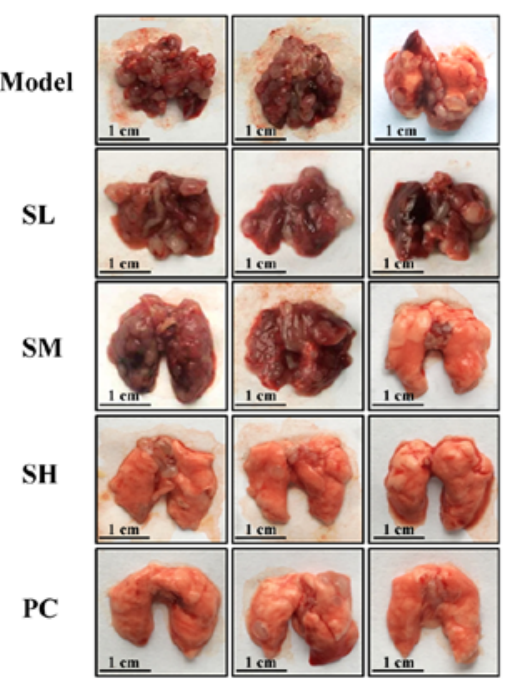

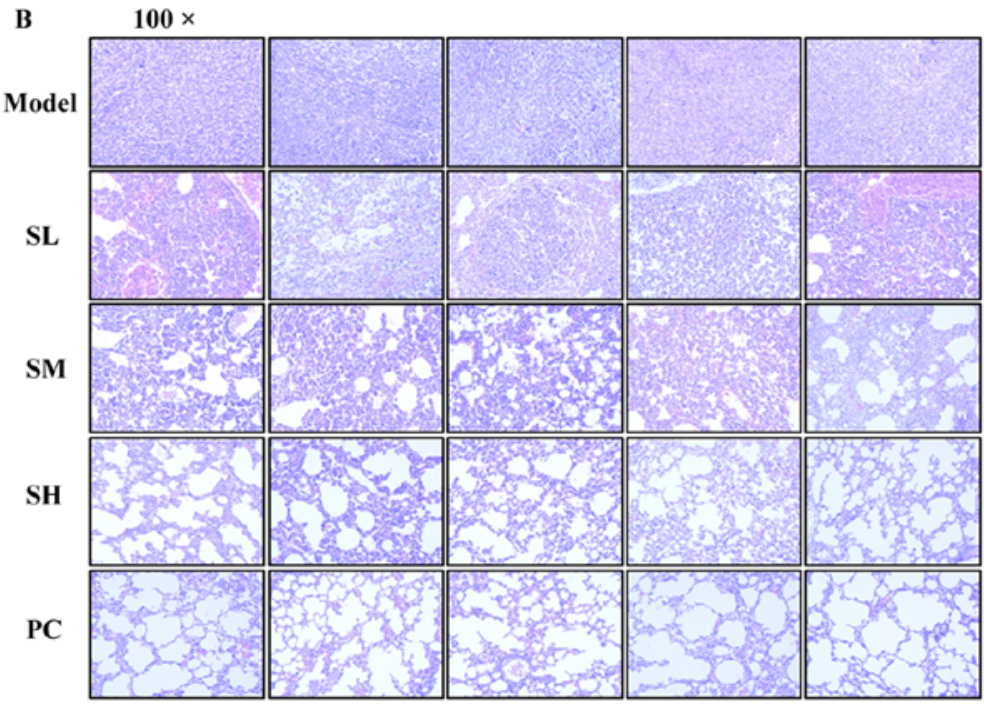
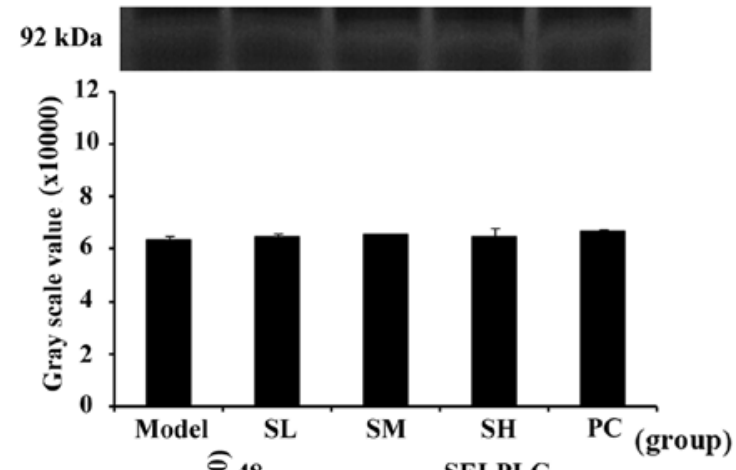

D

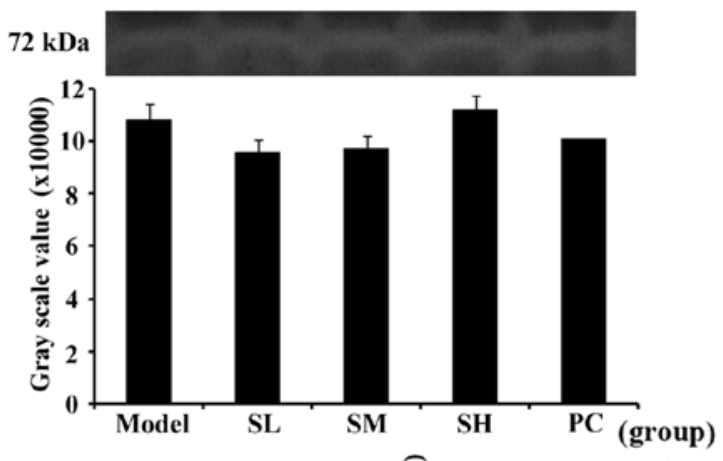

Platelet - $+++++\bigodot_{48}+$ SELP

SET $(\mu \mathrm{g} / \mathrm{ml}) \quad-\quad-\quad 20 \quad 40 \quad 80 \quad-\quad \oint^{4}$

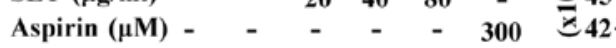

SELP

SELPLG

GPVI

ITGA2B

GAPDH
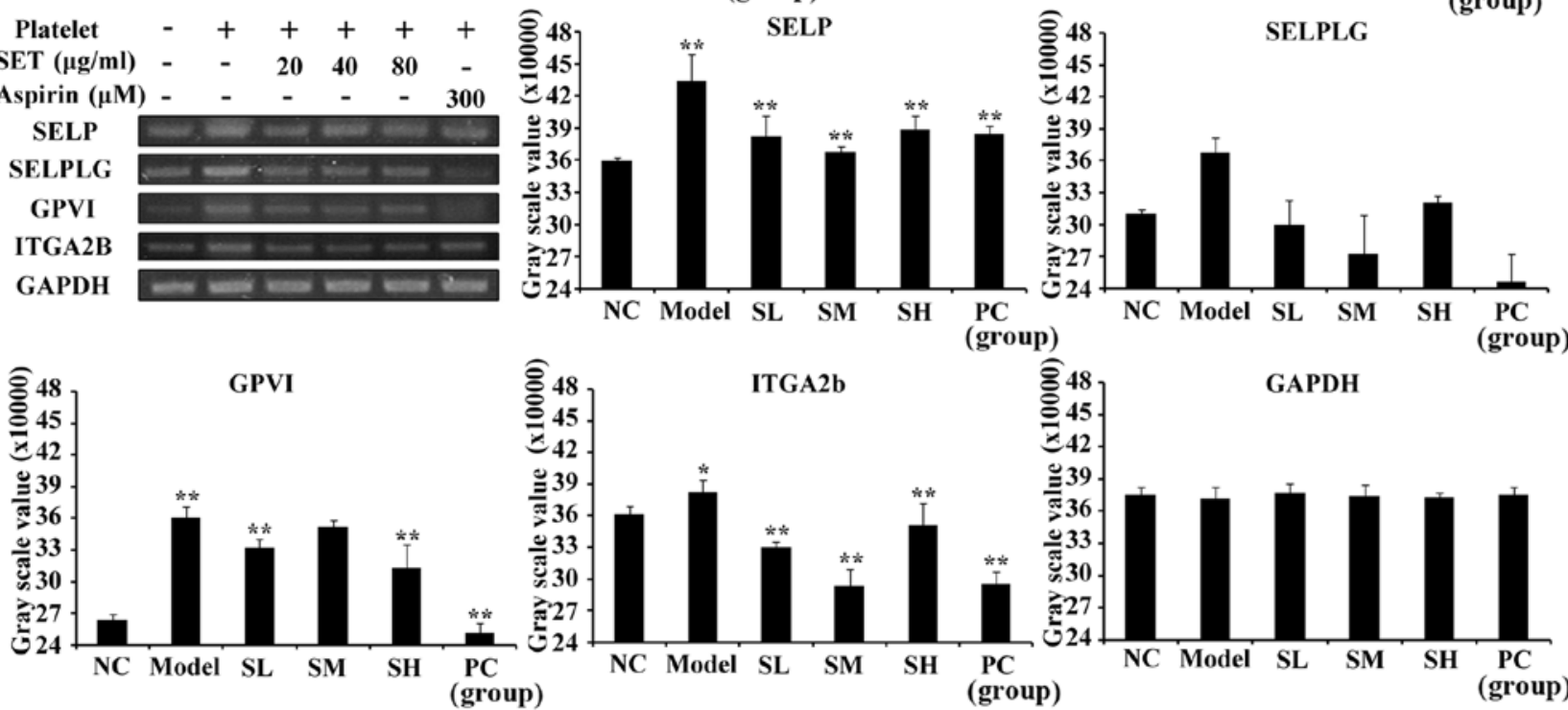

Figure 5. The 80\% ethanol extracts of Caulis Spatholobi (SET) suppressed the tumor pulmonary metastasis by certain mechanism for inhibition of tumor cell-induced platelet aggregation (TCIPA). (A) The general observation in lungs. The results showed that, with the increased dosage of SET treatment, quantity of lung metastatic sites decreased notably. (B) Tissue slices samples prepared from (A) were stained by hematoxylin and eosin (H\&E) for analyzing the metastatic intensity. The photo was taken by microscope at x100 magnification. (C) Gray scale values of matrix metalloproteinase (MMP)-2 and -9 were measured by zymography assay. It showed that there was no difference between each group. (D) The expression of TCIPA-related molecules were detected by RT-PCR assay. It indicated that SET exerts no regulation on selectin P ligand (SELPLG) expression. However, SET could downregulate the selectin P (SELP), glycoprotein VI (GPVI), integrin $\alpha 2 \mathrm{~b}$ (ITGA2B) expression which were stimulated via TCIPA. ${ }^{* *} \mathrm{P}<0.01$.

can be pathologically strengthened by certain tumor cells, resulting in blood hypercoagulability (24). On the other hand, tumor cell-induced hypercoagulable state provides an adaptive internal environment for facilitating the tumor cell escape from immune attack and shear stress accelerating hematogenous metastasis (25).
In 1973, Gasic et al first proposed the interaction between platelet and tumor cell through the in vitro culture of tumor cell-induced platelet activation (26). A platelet, as a vital factor for hypercoagulable state, promote metastasis and aggravate the malignant process (27). Accordingly, braking the collusion between the platelet and the tumor cell, tumor cell lose 
its shelter, then metastasis will be suppressed effectively. Fortunately, anticoagulant medication has been identified effective on certain cancers.

Taking ASA as an example, since 1990s, ASA has been used to be an antithrombotic therapy as a platelet inhibitor, treating and protecting from cancer-related thrombosis. Definitely, initial study shows that daily ASA reduces the long-term risk of several cancers and decreases the probability of distant metastasis (28). It is confirmable that TCIPA inhibition of treatment is able to have a favorable function on cancer progress, especially on metastasis (29). A meta-analysis evaluated that daily low-dose ASA as the anti-platelet therapy decreased the risk of adenocarcinomas with distant metastasis (30).

Although ASA was one of effective drugs on the inhibition of TCIPA, the deficiencies of clinical medication appeared gradually. For instance, emergence of drug resistance and reduction of drug susceptibility were severe with ASA as a single drug in metastatic treatment (31). In order to overcome this, new drugs are essential for anti-platelet therapy.

We focused on the novel extract from Caulis Spatholobi. As a traditional anticoagulant drug, Caulis Spatholobi has been used for improving hypercoagulable state and inhibiting the thrombosis in Chinese medicine. Initially, irregular menstruation, dysmenorrhea and rheumatic arthralgia were the typical scope of treatment for Caulis Spatholobi (32). In addition, various drug efficacies of Caulis Spatholobi have been found and determined including the antiviral, improvement of hematological system and antitumor activities $(33,34)$.

In our study, through MTT assay and ADP-induced platelet aggregation test, we determined the optimized time and dosage of drug treatment. After our efforts, we had discovered the efficacy of anti-platelet aggregation. By using confocal microscope, and flow cytometry analysis, the pathological activation of TCIPA could be observed clearly. SET was able to dissociate the aggregation by blocking the interaction of platelets and tumor cells. Moreover, the anti-metastasis efficacy of SET was also measured by mouse tail vein injection model in vivo. The metastasis was efficiently blocked by SET through suppressing the process of TCIPA. Molecularly, zymography assay proved that the anti-TCIPA efficacy of SET was regulated MMP-2 and -9-independently. In the presence of SET, the expressions of some adhesive molecules were downregulated, which indicates the preliminary molecular target of SET in TCIPA. Above all, by such mechanism, SET successfully dissociates the T-P complex and as a result, attenuates vascular transportation and dissemination of metastatic breast cancer cells.

Future work should pay more attention to two aspects of the study. Firstly, the material basis and the active chemical compound analysis of SET remain unclear. Therefore, further detailed separation, extraction, purification and identification studies are certainly necessary. Secondly, the anti-TCIPA efficacy of SET is a complicated pharmacological process, in which, the exact pathway and mechanism of SET remain to be further revealed. Overall, our study provided convincing evidence as well as practical approach for clinical chemotherapy, especially metastatic therapy, targeting TCIPA in the future.

\section{Acknowledgements}

This study was supported by the National Natural Science Foundation of China (grant no. 81303272).

\section{References}

1. DeSantis CE, Fedewa SA, Goding Sauer A, Kramer JL, Smith RA and Jemal A: Breast cancer statistics, 2015: Convergence of incidence rates between black and white women. CA Cancer J Clin 66: 31-42, 2016.

2. Baselga J, Cortés J, Kim S-B, Im SA, Hegg R, Im YH, Roman L, Pedrini JL, Pienkowski T, Knott A, et al; CLEOPATRA Study Group: Pertuzumab plus trastuzumab plus docetaxel for metastatic breast cancer. N Engl J Med 366: 109-119, 2012.

3. Wood SL, Pernemalm M, Crosbie PA and Whetton AD: The role of the tumor-microenvironment in lung cancer-metastasis and its relationship to potential therapeutic targets. Cancer Treat Rev 40: 558-566, 2014.

4. Johnson SA (ed): The Circulating Platelet. Elsevier, New York, NY, 2012.

5. Byzova TV, Kerr BA, Feng W and McCabe P: Platelets and the tumor cell microenvironment. Blood 122: SCI-32 - SCI-32, 2013.

6. Riedl J, Pabinger I and Ay C: Platelets in cancer and thrombosis. Hamostaseologie 34: 54-62, 2014.

7. Li N: Platelets in cancer metastasis: To help the 'villain' to do evil. Int J Cancer 138: 2078-2087, 2015.

8. Quail DF and Joyce JA: Microenvironmental regulation of tumor progression and metastasis. Nat Med 19: 1423-1437, 2013.

9. Goubran HA, Burnouf T, Radosevic M and El-Ekiaby M: The platelet-cancer loop. Eur J Intern Med 24: 393-400, 2013.

10. Goubran HA, Stakiw J, Radosevic $\mathrm{M}$ and Burnouf $\mathrm{T}$ : Platelet-cancer interactions. Semin Thromb Hemost 40: 296-305, 2014.

11. Stockert JC, Blázquez-Castro A, Cañete M, Horobin RW and Villanueva A: MTT assay for cell viability: Intracellular localization of the formazan product is in lipid droplets. Acta Histochem 114: 785-796, 2012.

12. Bentz J, O'Connor MP, Bednarczyk D, Coleman J, Lee C, Palm J, Pak YA, Perloff ES, Reyner E, Balimane P, et al: Variability in P-glycoprotein inhibitory potency $\left(\mathrm{IC}_{50}\right)$ using various in vitro experimental systems: Implications for universal digoxin drug-drug interaction risk assessment decision criteria. Drug Metab Dispos 41: 1347-1366, 2013.

13. Frenette PS, Johnson RC, Hynes RO and Wagner DD: Platelets roll on stimulated endothelium in vivo: An interaction mediated by endothelial P-selectin. Proc Natl Acad Sci USA 92: 7450-7454, 1995.

14. Angiolillo DJ, Fernández-Ortiz A, Bernardo E, Ramírez C, Sabaté M, Bañuelos C, Hernández-Antolín R, Escaned J, Moreno R, Alfonso F, et al: High clopidogrel loading dose during coronary stenting: Effects on drug response and interindividual variability. Eur Heart J 25: 1903-1910, 2004.

15. Shou L-M, Zhang Q-Y, Li W, Xie X, Chen K, Lian L, Li ZY, Gong FR, Dai KS, Mao YX, et al: Cantharidin and norcantharidin inhibit the ability of MCF-7 cells to adhere to platelets via protein kinase $C$ pathway-dependent downregulation of $\alpha 2$ integrin. Oncol Rep 30: 1059-1066, 2013.

16. Labelle M, Begum S and Hynes RO: Platelets guide the formation of early metastatic niches. Proc Natl Acad Sci USA 111: E3053-E3061, 2014.

17. Zhang WC, Shyh-Chang N, Yang H, Rai A, Umashankar S, Ma S, Soh BS, Sun LL, Tai BC, Nga ME, et al: Glycine decarboxylase activity drives non-small cell lung cancer tumor-initiating cells and tumorigenesis. Cell 148: 259-272, 2012.

18. Medina C, Harmon S, Inkielewicz I, Santos-Martinez MJ, Jones M, Cantwell P, Bazou D, Ledwidge M, Radomski MW and Gilmer JF: Differential inhibition of tumour cell-induced platelet aggregation by the nicotinate aspirin prodrug (ST0702) and aspirin. Br J Pharmacol 166: 938-949, 2012.

19. van Dongen JJ, Macintyre EA, Gabert JA, Delabesse E, Rossi V, Saglio G, Gottardi E, Rambaldi A, Dotti G, Griesinger F, et al: Standardized RT-PCR analysis of fusion gene transcripts from chromosome aberrations in acute leukemia for detection of minimal residual disease. Report of the BIOMED-1 Concerted Action: Investigation of minimal residual disease in acute leukemia. Leukemia 13: 1901-1928, 1999. 
20. Liu T, Tang H, Lang Y, Liu M and Li X: MicroRNA-27a functions as an oncogene in gastric adenocarcinoma by targeting prohibitin. Cancer Lett 273: 233-242, 2009.

21. Li M, Xing S, Zhang H, Shang S, Li X, Ren B, Li G, Chang X, $\mathrm{Li}$ Y and Li W: A matrix metalloproteinase inhibitor enhances anti-cytotoxic T lymphocyte antigen-4 antibody immunotherapy in breast cancer by reprogramming the tumor microenvironment. Oncol Rep 35: 1329-1339, 2016.

22. Gay LJ and Felding-Habermann B: Contribution of platelets to tumour metastasis. Nat Rev Cancer 11: 123-134, 2011.

23. Sørensen HT, Mellemkjaer L, Steffensen FH, Olsen JH and Nielsen GL: The risk of a diagnosis of cancer after primary deep venous thrombosis or pulmonary embolism. N Engl J Med 338 : 1169-1173, 1998

24. Donati MB, Gambacorti-Passerini C, Casali B, Falanga A, Vannotti P, Fossati G, Semeraro N and Gordon SG: Cancer procoagulant in human tumor cells: Evidence from melanoma patients. Cancer Res 46: 6471-6474, 1986.

25. Kannagi R: Carbohydrate-mediated cell adhesion involved in hematogenous metastasis of cancer. Glycoconj J 14: 577-584, 1997.

26. Gasic GJ, Gasic TB, Galanti N, Johnson T and Murphy S: Platelet-tumor-cell interactions in mice. The role of platelets in the spread of malignant disease. Int J Cancer 11: 704-718, 1973.

27. Falanga A: Thrombophilia in cancer. Semin Thromb Hemost 31 : 104-110, 2005
28. Rothwell PM, Wilson M, Price JF, Belch JF, Meade TW and Mehta Z: Effect of daily aspirin on risk of cancer metastasis: A study of incident cancers during randomised controlled trials. Lancet 379: 1591-1601, 2012.

29. Mitrugno A, Williams D, Kerrigan SW and Moran N: A novel and essential role for Fc $\gamma$ RIIa in cancer cell-induced platelet activation. Blood 123: 249-260, 2014.

30. Patrignani P, Filabozzi P and Patrono C: Selective cumulative inhibition of platelet thromboxane production by low-dose aspirin in healthy subjects. J Clin Invest 69: 1366-1372, 1982

31. Hankey GJ and Eikelboom JW: Aspirin resistance. Lancet 367: 606-617, 2006.

32. Wagner H, Bauer R, Melchart D, Xiao P-G and Staudinger A (eds): Caulis Spatholobi-Jixueteng. In: Chromatographic Fingerprint Analysis of Herbal Medicines Volume III. Vol 3. 1st edition. Springer, Switzerland, pp235-242, 2014.

33. Yan LG, Ruan JS, Zhang L, Fan FT, Zhang F, Wang AY, Zheng SZ, Zeng L, Li WL and Lu Y: Effect of aqueous extracts of several kinds of herbs on human platelet aggregation and expression of P-selectin in vitro. Chin J Integr Med 21: 286-290, 2015.

34. Liu B, Liu J, Chen J, Zhu D, Zhou H and Wang X: A study on anticancer activity of Caulis Spatholobi extract on human osteosarcoma Saos-2 cells. Afr J Tradit Complement Altern Medicines 10: 256-260, 2013. 